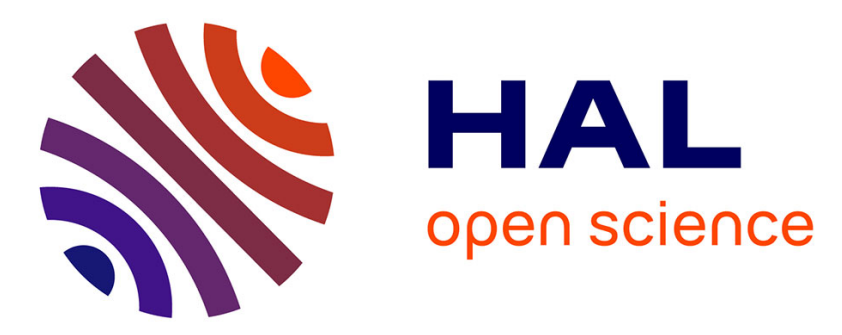

\title{
Two Wheeled Vehicle Dynamics Synthesis for Real-Time Applications
}

Hima Salim, Hichem Arioui

\section{To cite this version:}

Hima Salim, Hichem Arioui. Two Wheeled Vehicle Dynamics Synthesis for Real-Time Applications. 1st Mediterranean Conference on Intelligent Systems and Automation (CISA 08), Jun 2008, ANNABA, Algeria. pp.47-52, 10.1063/1.2953028 . hal-00420878

\section{HAL Id: hal-00420878 https://hal.science/hal-00420878}

Submitted on 2 Oct 2009

HAL is a multi-disciplinary open access archive for the deposit and dissemination of scientific research documents, whether they are published or not. The documents may come from teaching and research institutions in France or abroad, or from public or private research centers.
L'archive ouverte pluridisciplinaire HAL, est destinée au dépôt et à la diffusion de documents scientifiques de niveau recherche, publiés ou non, émanant des établissements d'enseignement et de recherche français ou étrangers, des laboratoires publics ou privés. 


\title{
Two Wheeled Vehicle Dynamics Synthesis for Real-Time Applications
}

\author{
Salim Hima* and Hichem Arioui * \\ * Informatics, Integrative Biology and Complex Systems CNRS-FRE 3190, 40 \\ Rue de Pelvoux, 91020 Evry Cedex, France. \\ e-mail: \{Salim.Hima, Hichem.Arioui\}@ibisc.univ-evry.fr.
}

\begin{abstract}
In this paper, we present a modeling technique for deriving the motorcycles equation of motion. The proposed technique is based on the recursive Newton-Euler approach and adapted to tree structure with floating base multibody systems. The derived model presents a low number of arithmetic operations, and hence, suitable for implementation into a two wheeled vehicles real-time applications such as driving simulators. The synthesized model takes in consideration the main wrenches that affect the behavior of motorcycle such as: pneumatic, aerodynamic, suspensions, contact constraints and control inputs.
\end{abstract}

Keywords: Motorcycle driving simulator, motorcycle dynamics, Recursive Newton-Euler algorithm.

\section{INTRODUCTION}

$\mathbf{I}$ $\mathrm{N}$ the two last decades, the safety of ground vehicles has known a significant progress. Due to the reduction of the electronics and the informatics equipments, several intelligent systems have been developed to optimize the performance of the ground vehicles. In general, the development of such systems is based on the vehicle behavioral model that allows the development and testing them without need for real prototypes. In such applications, real-time constraint is not crucial. However, this constraint become important when the application is centered around a human operator such as driving simulators.

In fact, driving simulators have initially proposed in aeronautic field to train a novice pilots, enhance the performance of experimental ones and also testing a new systems and designs in a safe environment and without need of a real aircraft. Few years later, this technology has been extended to four wheeled vehicles field and recently to two wheeled vehicles field.

The idea behind this technology is to immerse the rider in a virtual traffic environment by properly exciting his perception organs with a convenient cues. In literature, we distinguish two type of driving simulators: a fixed base and motion base simulators. The former can merely provide the rider with a visual and auditive cues, while the second add an appropriate acceleration cue component by moving the simulator motionbase, on which the rider is rising, within its limited workspace.

One of the central components of driving simulator is a vehicle's behavioral model. When the driver acts on the vehicle control inputs, these actions are sensed and transmitted to the vehicle behavioral model bloc. This bloc computes the state and accelerations of the vehicle and transmit them to different simulator components (visual, auditive, and motion-base) in order o update their states. It is important from perception point of view to reduce the delay time between driver actions instant and the updating the whole simulation component states instant.

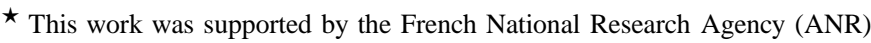
in the Framework of SIMCOM project.
In the framework of the SIMACOM project, a new motorcycle motion-base simulator has been designed, see Nehaoua [2007]. The proposed design is customized to reproduce the relevant motorcycle situations in real driving. In this paper, we are interested to derive the motorcycle behavioral model with a low arithmetic operation in order to reduce the computation time.

In literature, several model are proposed in the scope of control purposes. These models are partial and focused on a local behaviors and neglect some element such as suspension or road irregularity. However, driving simulator need for a broad model in order to cover a very large spectrum of rider's actions.

Multibody mechanics offers a convenient framework to derive a motorcycle behavioral model. In Cossalter [2002] authors have adopted Lagrange formalism to derive their motorcycle motion model. A direct application of this approach, leads to unattractive performances in term of number of operations and implementation facilities. An algorithmic formulation alternative of this formalism, that presents the advantage to be easily debugged and easy to implement, has been presented in Hima [2007].

Exploiting the recursive scheme in computing some kinematic quantities, very efficient algorithms have been proposed to solve the inverse dynamics of open chain multibody systems, see Featherstone [2000], Khalil [1987], Hollerbach [1980]. So, the direct dynamics can be derived from the inverse dynamics using the articulated body algorithm (ABA), Featherstone [2000]. This algorithm is known to be fast, accurate and stable Khalil [1999].

In this paper, we extend ABA algorithm to derive a fast direct model of motorcycles suitable for real-time application such as two wheeled interactive simulator. In the second section of this paper, a brief presentation of our simulator is given. The third Section is devoted to present the derivation steps of motorcycle forward dynamics. In the fourth section, simulation results is illustrated. Finally, conclusion remarks and future works are outlined. 


\section{SIMACOM PROJECT PRESENTATION}

The primary goal of SIMACOM project is to propose a low cost replicable motorcycle simulator. It's dedicated to driving schools for training a novice riders and perform the experienced riders aptitudes face to life-threatening situations in a safe environment. Mainly, the proposed simulator architecture is designed around five subsystems: a visual subsystem, acoustic subsystem, motion-base platform, motorcycle model and haptic subsystems, see Fig 1. The visual subsystem allows the immersion of the rider in a virtual traffic environment, managed by ARCHISIM software, and provides him with velocity cue.

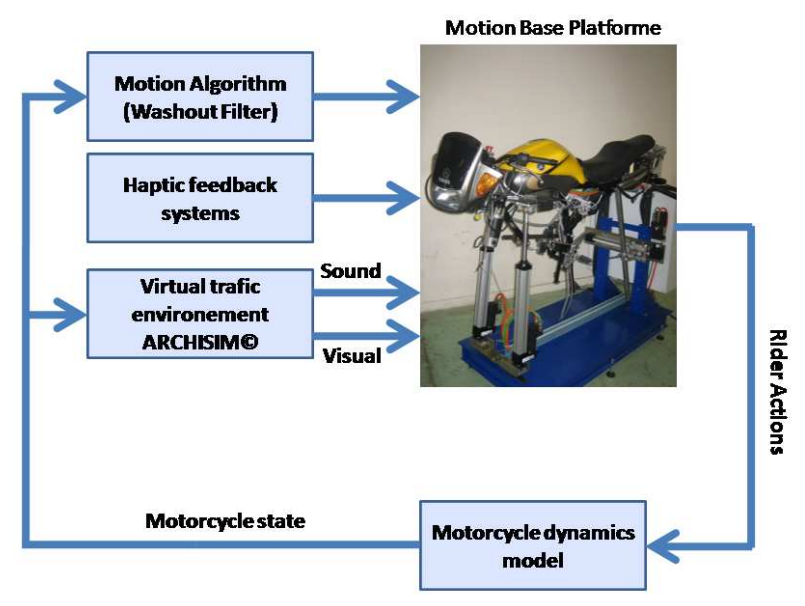

Fig. 1. SIMACOM project simulator architecture

In Panerai [2001] the authors have stated that, an acoustic cues deprivation leads to a systematic increase tendency of the speed by drivers. To prevent this problem, and making the simulation more realistic, an acoustic subsystem is placed to reproduce sounds resulting from the juxtaposition of many sources where the main ones come from the engine and tires. In addition, inertial cues are generated by stimulating the rider vestibular organ by moving him with a proper motions. For this aim, a motion-base platform, which consists of a real motorcycle structure hanged on a parallel robot, is designed to reproduce the main motorcycle situations such as bending in braking situation, leaning in cornering situation and also the rear tire skidding situation. That's why, two prismatic actuator are placed in the front and connected to saddle body by way of spherical joints. So, the pitch and roll of the motion-base are directly controlled by changing the legs lengths using two DC motors. In addition, illusion of rear tire skidding is created by placing a sliding system at the rear of the motion-base to perform a horizontal displacement, see Fig 2.

Changes in previous described subsystems states, are made in response to the rider actions captured by placing a sensors in the motorcycle control organs (throttle angle, braking and clutch leavers). Once rider's action are sensed, they are sent to motorcycle dynamics model to compute motorcycle's motion (configuration, velocities and accelerations). These informations are then provided to visual and acoustic subsystem to update the virtual environment and the adequate sounds. In the same time, motion informations are also provided to washout filter in order to transform it into motions compatible with motion-base workspace limitations.

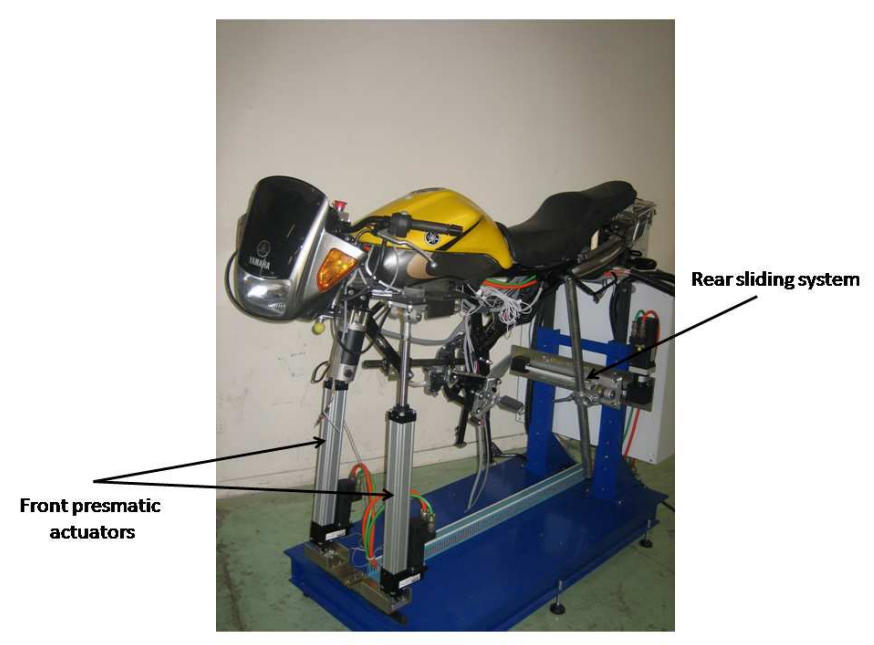

Fig. 2. Motion-base platform prototype

When riding a motorcycle, the ground-tire interaction forces and the gyroscopic precession force of the front wheel are transmitted to handlebar and so felt by the rider. Furthermore, tension forces exerted on the rider arms in acceleration and deceleration phases, caused by the rider bust inclination, are present. In order to reproduce these forces and make the simulator more efficient, two haptic feedback subsystems are integrated to our simulator (Fig 3). The first subsystem consists of a DC motor connected to the steering axis by way of pulley-belt system. It permits the application of a torque on the steering axis to give the rider sensation of the tire-ground and gyroscopic forces. This torque is computed from the motorcycle dynamics model. Moreover, the second subsystem consists of a mechanism that endow the handlebar with a longitudinal translation motion controlled by a brushless actuator, see Fig 3. In fact, in acceleration phase, the handlebar translates far from the rider and hence, pulls the rider arms to create illusion of the bust inclination away from the handlebar. Otherwise, in deceleration phase, handlebar translates toward the rider and so, push the rider arms to create illusion of the bust inclination toward handlebar.

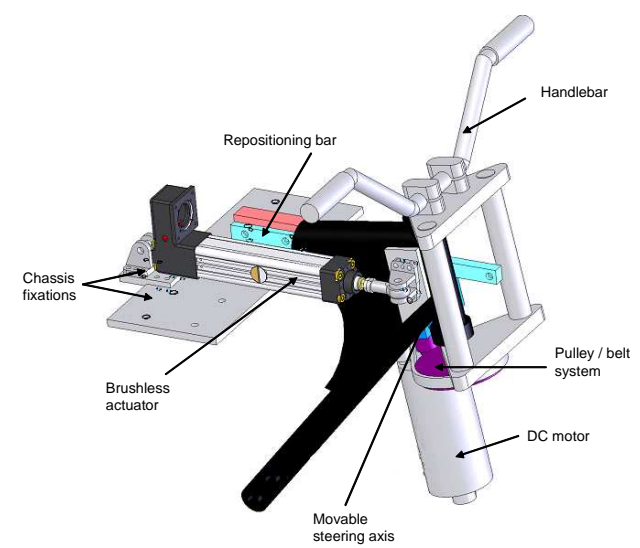

Fig. 3. Haptic feedback systems in the handlebar

For further description of this simulator and kinematic study of the motion-base, readers can consult Nehaoua [2007]. 


\section{MOTORCYCLE MODELLING}

Motorcycle direct dynamics can be derived using mechanics of multibody systems theory. Mainly, the motorcycle is composed by the saddle body, the front upper part (handlebar and upper part of the front suspension), the front lower part of the front suspension, the swinging arm and finally the front and rear tires. All these parts are connected between them with a simple joints. the Handlebar and the swinging arm are attached to saddle body by a simple rotoid joints. The font lower part is linked to the front upper part by prismatic joint. In addition, the rear suspension is connected to saddle with a rotoid joint from one side and to the swinging arm with a rotoid joint also from the other side. This creates a closed kinematic loop, and so, leads to a modelling difficulties. Finally, rear and front wheels are respectively connected to the other tips of swinging arm and front lower part as illustrated in Fig 4.

The motion of the motorcycle is referenced to an inertial frame $\mathcal{R}_{0}\left(O_{0}, x_{0}, y_{0}, z_{0}\right)$. Moreover, to each body $B_{i}$ an orthonormed frame is rigidly attached. In order to completely define the configuration of the motorcycle, a set of 11 DOFs have been considered. A full characterization of motorcycle's situation can be done by the position and orientation of the saddle $(x, y, z, \phi, \theta, \psi)$, the steering angle or handlebar orientation, $\delta$, the elongation of the front suspension, $L$, the rotation angle of the swinging arm with respect to the saddle, $\theta_{s}$ (elongation of the rear suspension), and finally, the rotation angles of the front and rear tires, $\theta_{f}$ and $\theta_{r}$.

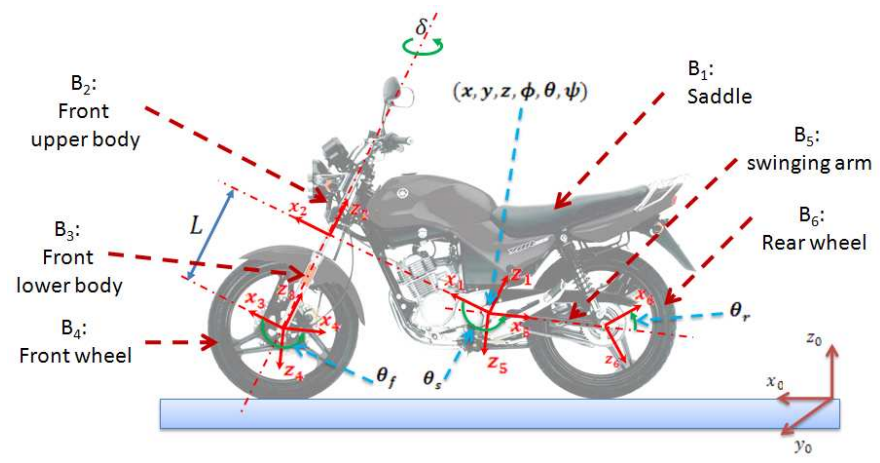

Fig. 4. Motorcycle configuration parametrization

\subsection{Direct Dynamics}

Application of the classical formalisms from mechanics of multiboby systems can leads to an inefficient dynamic models in term of the number of arithmetic operations. However, recursive techniques present another alternative to reduce greatly the computational burden and that should allow a real-time computation of the direct dynamics of the motorcycle. In Khalil [1999] and Hollerbach [1980], Recursive Newton-Euler Algorithm, RENA, have been shown to be the fast one. Originally, this technique is developed to solve the inverse dynamic model of the open chain manipulator with a fixed base for control purposes. By projecting the dynamics of each body in its attached frame, the acceleration of the joint variable can be easily derived without need to compute the total inertia matrix of the whole system and invert it. In addition, this technique, named Articulated Body Algorithm (ABA), is more numerically stable then inertia matrix inversion method, see Khalil [1999].

To illustrate the ABA, consider the multibody system sketched in Fig 5. Let $\mathbb{V}_{i}=\left(v_{i}^{T}, \omega_{i}^{T}\right)^{T}$ be the twist vector of $B_{i}$ where $v_{i}$ is its linear velocity and $\omega_{i}$ is its angular velocity. $\mathbf{k}_{i}$ is the $i^{\text {th }}$ joint axis, $\mathbb{F}^{e_{i}}=\left(F^{e_{i} T}, M^{e_{i} T}\right)^{T}$ is the external wrench acting on $B_{i}, \mathbf{L}_{i}$ the position vector of $(i+1)^{\text {th }}$ joint origin with respect to $i^{\text {th }}$ joint origin, $\mathbf{S}_{i}$ is the position vector of the $B_{i}$ center of gravity with respect to the $i^{\text {th }}$ joint origin, ${ }^{i} \mathbf{R}_{i-1}$ the transformation matrix from $B_{i-1}$ to $B_{i}, \mathbb{F}_{i}=\left(F_{i}^{T}, M_{i}^{T}\right)^{T}$ is the link wrench vector transmitted to $B_{i}$ from $B_{i-1}, m_{i}$ and $\mathbf{J}_{i}$ are, respectively, the mass and the inertia tensor of the body $B_{i}, q_{i}$ is $i^{\text {th }}$ joint variable and $\tau_{i}$ be the actuator force/torque acting on the joint $i$. In the sequel of this paper, the expression ${ }^{i} \mathbf{X}_{j}$ means that the vector $\mathbf{X}_{j}$ is projected into the frame associated to $B_{i}$.

Let $\sigma_{i}$ be a binary variable defining the joint type and given by:

$$
\sigma_{i}= \begin{cases}1 & \text { if joint } \mathrm{i} \text { is a prismatic } \\ 0 & \text { if joint } \mathrm{i} \text { is a revolute }\end{cases}
$$

Let ${ }^{i} \mathbb{T}_{i-1}$ be a $6 \times 6$ matrix defined as:

$$
{ }^{i} \mathbb{T}_{i-1}=\left(\begin{array}{cc}
{ }^{i} \mathbf{R}_{i-1} & -{ }^{i} \mathbf{R}_{i-1}{ }^{i-1} \tilde{\mathbf{L}}_{i-1} \\
0_{3 \times 3} & { }^{i} \mathbf{R}_{i-1}
\end{array}\right)
$$

where $\sim$ is the skew-symmetric matrix operator for a vector $\mathbf{v}=(x, y, z)^{T}$ defined by:

$$
\tilde{\mathbf{v}}=\mathbf{v} \times=\left(\begin{array}{ccc}
0 & -z & y \\
z & 0 & -x \\
-y & x & 0
\end{array}\right)
$$

Let ${ }^{i} \mathbb{J}_{i}$ be the global inertia matrix of $B_{i}$ given by:

$$
{ }^{i} \mathbb{J}_{i}=\left(\begin{array}{cc}
m_{i} \mathbf{I}_{3 \times 3} & -m_{i}{ }^{i} \tilde{\mathbf{S}}_{i} \\
m_{i}{ }_{i} \tilde{\mathbf{S}}_{i} & \mathbf{J}_{i}
\end{array}\right)
$$

and finally let ${ }^{i} \mathbf{a}_{i}=\left[\begin{array}{ll}\sigma_{i}{ }_{i} \mathbf{k}_{i}^{T} & \bar{\sigma}_{i}{ }^{i} \mathbf{k}_{i}^{T}\end{array}\right]^{T}$.

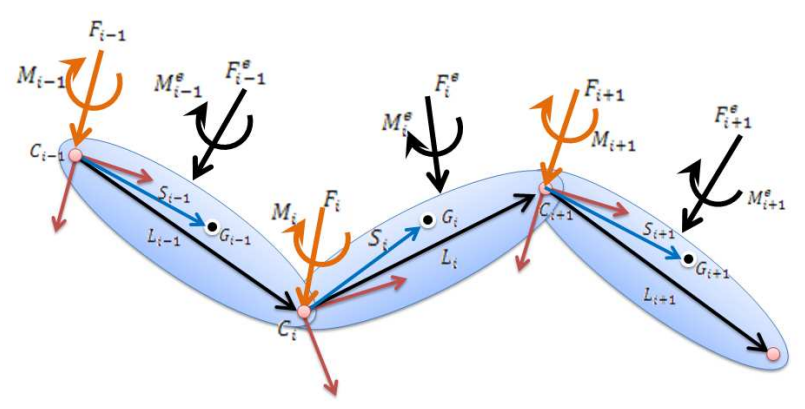

Fig. 5. Open chain multibody system

The ABA is based on three steps. In the first step (forward recursion), velocities, Coriolis, centrifuge and external forces are updated for each body. The second step (backward recursion), the total forces and inertia matrices felt by each joint are computed. in final step (forward recursion), the joints accelerations and dynamic torsors are computed for each body. Setting $\omega_{0}=\dot{\omega}_{0}=0$ and $\mathbb{V}_{0}=\left(-\mathbf{g}, 0_{1 \times 3}\right)^{T}$, where $\mathbf{g}$ is the gravity vector. So, the $\mathrm{ABA}$ is given by: 


\section{First Recursion}

$$
\begin{gathered}
\text { for } i=1 \rightarrow n \mathbf{d o} \\
\left.\qquad \begin{array}{c}
{ }^{i} \mathbb{V}_{i-1}={ }^{i} \mathbb{T}_{i-1}{ }^{i-1} \mathbb{V}_{i-1}+\dot{q}_{i}{ }_{i} \mathbf{a}_{i} \\
{ }^{i} \gamma_{i}=\left[\begin{array}{c}
{ }^{i} \mathbf{R}_{i-1}\left({ }^{i-1} \omega_{i-1} \times\left({ }^{i-1} \omega_{i-1} \times{ }^{i-1} \mathbf{L}_{i-1}\right)\right) \\
+2 \sigma_{i}\left({ }^{i} \omega_{i-1} \times \dot{q}_{i}{ }^{i} \mathbf{k}_{i}\right) \\
\bar{\sigma}_{i}\left({ }^{i} \omega_{i-1} \times \dot{q}_{i}{ }^{i} \mathbf{k}_{i}\right)
\end{array}\right] \\
{ }^{i} \beta_{i}={ }^{i} F_{i}^{e}-\left[\begin{array}{c}
{ }^{i} \omega_{i} \times\left({ }^{i} \omega_{i} \times m_{i}{ }^{i} \mathbf{S}_{i}\right) \\
{ }^{i} \omega_{i} \times\left({ }^{i} \mathbf{J}_{i}{ }^{i} \omega_{i}\right)
\end{array}\right]
\end{array}\right]
\end{gathered}
$$

end

\section{Second recursion}

$$
\begin{aligned}
& { }^{n} \beta_{n}^{*}={ }^{n} \beta_{n} \\
& { }^{n} \mathbb{J}_{n}^{*}={ }^{n} \mathbb{J}_{n} \\
& \text { for } i=n \rightarrow 1 \text { do } \\
& \left.\begin{array}{r}
{ }^{i-1} \beta_{i-1}^{*}={ }^{i-1} \beta_{i-1}-{ }^{i} \mathbb{T}_{i-1}^{T}\left[-{ }^{i} \beta_{i}^{*}\right. \\
\left.+{ }^{i} \mathbb{J}_{i}^{\star i} \gamma_{i}+\frac{{ }^{i} \mathbb{J}_{i}^{* i} \mathbf{a}_{i}\left\{{ }^{i} \mathbf{a}_{i}^{T}\left({ }^{i} \mathbb{J}_{i}^{* i} \gamma_{i}+{ }^{i} \beta_{i}^{*}\right)+\tau_{i}\right\}}{{ }^{i} \mathbf{a}_{i}^{T i} \mathbb{J}_{i}^{* i} \mathbf{a}_{i}}\right] \\
{ }^{i-1} \mathbb{J}_{i-1}^{*}={ }^{i-1} \mathbb{J}_{i-1}+{ }^{i} \mathbb{T}_{i-1}^{T}\left[{ }^{i} \mathbb{J}_{i}^{*}-\frac{{ }^{i}{ }_{i}^{* i} \mathbf{a}_{i}{ }^{i} \mathbf{a}_{i}^{T i} \mathbb{J}_{i}^{*}}{{ }^{i} \mathbf{a}_{i}^{T}{ }^{i} J_{i}^{*} \mathbf{a}_{i}}\right]
\end{array}\right]
\end{aligned}
$$

end

\section{Third recursion}

$$
\begin{aligned}
& \text { for } i=1 \rightarrow n \mathbf{d o} \\
& \qquad \begin{array}{l}
{ }^{i} \dot{\mathbb{V}}_{i-1}={ }^{i} \mathbb{T}_{i-1}{ }^{i-1} \dot{\mathbb{V}}_{i-1} \\
\ddot{q}_{i}=\mathbf{H}_{i}^{-1}\left[-{ }^{i} \mathbf{a}_{i}^{T i} \mathbb{J}_{i}^{*}\left({ }^{i} \dot{\mathbb{V}}_{i-1}+{ }^{i} \gamma_{i}\right)+\tau_{i}+{ }^{i} \mathbf{a}_{i}^{T i} \beta_{i}^{*}\right] \\
{ }^{i} \mathbb{F}_{i}=\left[\begin{array}{c}
{ }^{i} \mathbf{F}_{i} \\
{ }^{i} \mathbf{M}_{i}
\end{array}\right]={ }^{i} \mathbb{K}_{i}{ }^{i} \dot{\mathbb{V}}_{i-1}+{ }^{i} \alpha_{i} \\
{ }^{i} \dot{\mathbb{V}}_{i}={ }^{i} \dot{\mathbb{V}}_{i-1}+{ }^{i} \mathbf{a}_{i} \ddot{q}+{ }^{i} \gamma_{i}
\end{array}
\end{aligned}
$$

end

Algorithm 1. Recursive Newton-Euler algorithm for direct dynamics

Removing the rear suspension and replace it with two forces acting on the saddle and swinging arm at rear suspension fixation points, the motorcycle can be considered as a tree structure system with floating base. Hence, algorithm 1 is used to compute the accelerations of relative configurations $\left(\delta, L, \theta_{f}, \theta_{s}, \theta_{r}\right)$, and forces acting on their corresponding articulations for the open chains $\left(B_{2}, B_{3}, B_{4}\right)$ and $\left(B_{5}, B_{6}\right)$. The floating base (saddle) accelerations are often obtained by considering 6 virtual bodies linked between them by a single saddle's degree of freedoms. This leads to consider a serial chain with 6 bodies, and hence, apply RENA algorithm again. In our approach, and to reduce the number of arithmetic operations, we have isolated the saddle body only, and replace the front and the rear chains by their transmitted dynamical wrenches $\left(\mathbb{F}_{2}, \mathbb{F}_{5}\right)$ at the $2^{\text {nd }}$ and $5^{\text {th }}$ joints origins. In this case, we can consider the saddle as a spatial rigid body subjected to external forces. So, the saddle's accelerations are then given by:

$$
\begin{aligned}
{ }^{1} \dot{\mathbb{V}}_{1} & ={ }^{1} \mathbb{J}_{1}^{-1}\left(\mathbf{C}\left(\eta,{ }^{1} \mathbb{V}_{1}\right)+\mathbf{G}(\eta)+{ }^{1} \mathbb{F}_{2}+{ }^{1} \mathbb{F}_{5}+{ }^{1} \mathbb{F}_{r s}\right) \\
\dot{\eta} & =\mathbb{R}^{1} \mathbb{V}_{1}
\end{aligned}
$$

where $\eta=(x, y, z, \phi, \theta, \psi)$ is the configuration vector of the saddle. ${ }^{1} \mathbb{V}_{1}$ is the vector of the linear and angular velocities of saddle body with respect to its frame. ${ }^{1} \mathbb{J}_{1}$ is the inertia matrix of the saddle body expressed in its frame. C and $\mathbf{G}$ are, respectively, the Centrifuge/Coriolis and weight wrenches. $\mathbb{F}_{r s}$ is rear suspension force acting on the saddle. $\mathbb{R}$ is the transformation matrix form $B_{1}$ frame to inertial frame $\mathcal{R}_{0}$ and can be given by:

$$
\mathbb{R}=\left(\begin{array}{ccc}
c_{\psi} c_{\theta} & -s_{\psi} c_{\phi}+c_{\psi} s_{\theta} s_{\phi} & s_{\psi} s_{\phi}+c_{\psi} c_{\phi} s_{\theta} \\
s_{\psi} c_{\theta} & c_{\psi} c_{\phi}+s_{\phi} s_{\theta} s_{\psi} & -c_{\psi} s_{\phi}+s_{\theta} s_{\psi} c_{\phi} \\
-s_{\theta} & c_{\theta} s_{\phi} & c_{\theta} c_{\phi}
\end{array}\right)
$$

where $c_{x}=\cos (x)$ and $s_{x}=\sin (x)$.

\subsection{External forces}

In this section, we summarize the principal forces and moments affecting the motorcycle behavior.

Suspension forces In order to absorb the shocks caused by the road irregularities and bumps, the motorcycle is equipped with a rear and front suspension systems. In this paper a linearly dependence of the forces generated by the suspensions on the elongations and their rates of change is considered:

$$
\mathbf{F}_{i}=K_{i}\left(L_{i}-L_{i 0}\right)+C_{i} \dot{L}_{i}
$$

where $i \in\{r s, f s\}$ designates the rear or front suspension, $L_{i 0}$ is its free load elongation, $K_{i}$ is the suspension stiffness and $C_{i}$ is the damper coefficient. According to Fig 6, the suspension length can be expressed as:

$$
\begin{aligned}
L_{f s} & =\sqrt{\left({ }^{2} P_{f s_{u}}-{ }^{2} P_{f_{s_{l}}}\right)^{T}\left({ }^{2} P_{f_{s_{u}}}-{ }^{2} P_{f s_{l}}\right)} \\
L_{r s} & =\sqrt{\left({ }^{1} P_{r s_{u}}-{ }^{1} P_{r s_{l}}\right)^{T}\left({ }^{1} P_{r s_{u}}-{ }^{1} P_{r s_{l}}\right)}
\end{aligned}
$$

where $P_{i_{u}}$ and $P_{i_{l}}$ denote respectively the position of the upper and lower extremities of suspension $i$. Hence, the rate of change of suspensions lengths are given by:

$$
\begin{aligned}
& \dot{L}_{f s}=\frac{1}{L_{f s}}\left({ }^{2} \dot{P}_{f_{s_{u}}}-{ }^{2} \dot{P}_{f_{s_{l}}}\right)^{T}\left({ }^{2} P_{f_{s_{u}}}-{ }^{2} P_{f_{s_{l}}}\right) \\
& \dot{L}_{r s}=\frac{1}{L_{r s}}\left({ }^{1} \dot{P}_{r s_{u}}-{ }^{1} \dot{P}_{r s_{l}}\right)^{T}\left({ }^{1} P_{r s_{u}}-{ }^{1} P_{r_{l}}\right)
\end{aligned}
$$

Hence, the suspense wrenches are given by:

$$
\begin{aligned}
{ }^{1} \mathbb{F}_{r s_{u}}= & {\left[\frac{\left({ }^{1} P_{r s_{u}}-{ }^{1} P_{r s_{l}}\right)^{T}}{L_{r s}} \mathbf{F}_{r s},{ }^{1} P_{r s_{u}} \times \frac{\left({ }^{1} P_{r s_{u}}-{ }^{1} P_{r s_{l}}\right)^{T}}{L_{r s}} \mathbf{F}_{r s}\right]^{T} } \\
{ }^{5} \mathbb{F}_{r s_{l}} & =\left[\frac{\left({ }^{5} P_{r s_{l}}-{ }^{5} P_{r s_{u}}\right)^{T}}{L_{r s}} \mathbf{F}_{r s},{ }^{5} P_{r s_{l}} \times \frac{\left({ }^{5} P_{r s_{l}}-{ }^{5} P_{r s_{u}}\right)^{T}}{L_{r s}} \mathbf{F}_{r s}\right]^{T} \\
{ }^{2} \mathbb{F}_{f s_{u}}= & {\left[\frac{\left({ }^{2} P_{f_{s_{u}}}-{ }^{2} P_{f s_{l}}\right)^{T}}{L_{f s}} \mathbf{F}_{f s_{s}},{ }^{2} P_{f_{s_{u}}} \times \frac{\left({ }^{2} P_{f s_{u}}-{ }^{2} P_{f s_{l}}\right)^{T}}{L_{f s}} \mathbf{F}_{f s}\right]^{T} } \\
{ }^{3} \mathbb{F}_{f_{s_{l}}}= & {\left[\frac{\left({ }^{3} P_{f_{s_{l}}}-{ }^{3} P_{f_{s_{u}}}\right)^{T}}{L_{f s}} \mathbf{F}_{f s_{s}},{ }^{3} P_{f_{s_{l}}} \times \frac{\left({ }^{3} P_{f_{s_{l}}}-{ }^{3} P_{f_{s_{u}}}\right)^{T}}{L_{f s}} \mathbf{F}_{f s}\right]^{T} }
\end{aligned}
$$




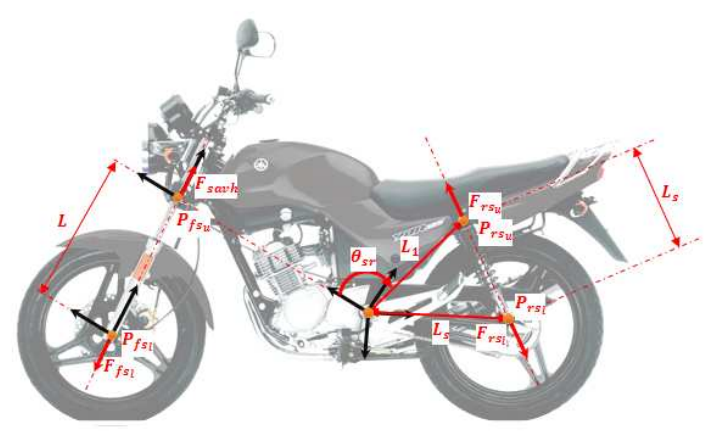

Fig. 6. Suspension systems wrenches

Tire/road contact wrench Behavior of the ground vehicles depends significantly on the nature of the interaction between the tire and the road. Indeed, in the last two decades, and due to the importance of this phenomena in the security of ground vehicles, several efforts are done to establish mathematical models characterizing the friction forces and moments of the tire/road contact. The well known and widely used one is Pacejka Magic formula. This model captures in steady-state motion, the tire/road forces and moments, in algebraic equations form, with respect to kinematic quantities, longitudinal slip $\kappa$ and lateral slip $\beta$, and the load. The steady-state behavior restriction still valid in the scope that, the effect of the tire dynamics is generally small compared to the effect of the complete motorcycle dynamics.

Initially, Pacejka model has been developed for four wheels vehicles. In this case, wheels stay approximately vertical and consequently, the camber angle can be neglected. Other varieties of this model have been proposed and adapted to motorcycle tires by including the camber angle. In fact, this angle could achieves $50^{\circ}$ for racing motorcycles when cornering and hence, could'nt be ignored, Lot [2004].

Among a six components of the tire/road contact wrench, we have considered the principal ones: longitudinal and lateral forces and the aligning moment, Sharp [2004], Pacejka [1997].

Aerodynamic wrench When a body moves in a fluid, this leads to the apparition of aerodynamic forces and moments proportional to the relative motion of the body with respect to the fluid, applied to the center of pressure. The most important components are the drag and the lift. In our developed motorcycle model we have consider these two forces. The drag force opposes the motion of the motorcycle, and hence limit the maximum achievable velocity and also acceleration performance. In otherwise, the lift force can have an undesirable effects on the motorcycle performances. Indeed, it reduces the load on the wheels and, thus, decreases the tire adherence. In general, the drag and the lift forces are expressed in a linear form with respect to the square of the motorcycle linear velocity relative to air:

$$
\begin{aligned}
F_{D} & =\frac{1}{2} C_{D} \rho S\left\|V_{a}\right\|^{2} \\
F_{L} & =\frac{1}{2} C_{L} \rho S\left\|V_{a}\right\|^{2}
\end{aligned}
$$

where $C_{D}$ and $C_{l}$ are dimensionless aerodynamic coefficient, $\rho$ is the air density. $V_{a}=V-V_{\infty}$ is motorcycle relative velocity where $V$ is the motorcycle velocity and $V_{\infty}$ is the air velocity. $S$ is the frontal motorcycle area exposed to the air flow.

Contact constraints forces Keeping the tires in contact with the road imposes two constraints. In order to satisfy these constraints we have placed two spring-dapper systems between the road and the tires contact points. This formulation is more advantageous than using Lagrangian multipliers which need to include constraints stabilizers to prevent constraints divergence and also can capture carcasses elasticity features.

Input controls The last considered external forces acting on the motorcycle behavior coming from the rider actions through the engine torque, brakes torques and finally steering torque. We have modeled these actions by a pure torque and we don't integrate their dynamic models, which will be considered in future works.

\section{SIMULATION RESULTS}

In the lack of a wide controller to handle the whole motorcycle behavior, we have synthesized a basic autopilot based on a linear PID controller for motorcycle velocity regulation in street forward motion. In this simulation we have modeled the engine by a first order filter to take into account the delay of the engine reaction. The delivered torque by the engine is then bounded by the maximum allowable torque. The resultant torque is applied at the rear tire axis. To test the longitudinal behavior of the developed motorcycle, we have considered a scenario with three phases, acceleration phase up to $15 \mathrm{~m} / \mathrm{s}$, a second acceleration phase up to $30 \mathrm{~m} / \mathrm{s}$, and finally a deceleration phase down to $20 \mathrm{~m} / \mathrm{s}$, see Fig 7 . In this figure, the steady error between the desired and the motorcycle errors in the three phases are different. It is most important when the motorcycle travels with a high speed. The origin of this effect is related to the aerodynamic drag force which is proportional to the square of the motorcycle speed. In Fig 8, requested autopilot control torque and effective control torque delivered by the engine at the rear tire axis are sketched. The effect of the engine delay and torque limitation can be observed on the convergence time of the speed profile. Fig 9 illustrates the effective motorcycle driving forces generated by the rear and the front tires pneumatic models.

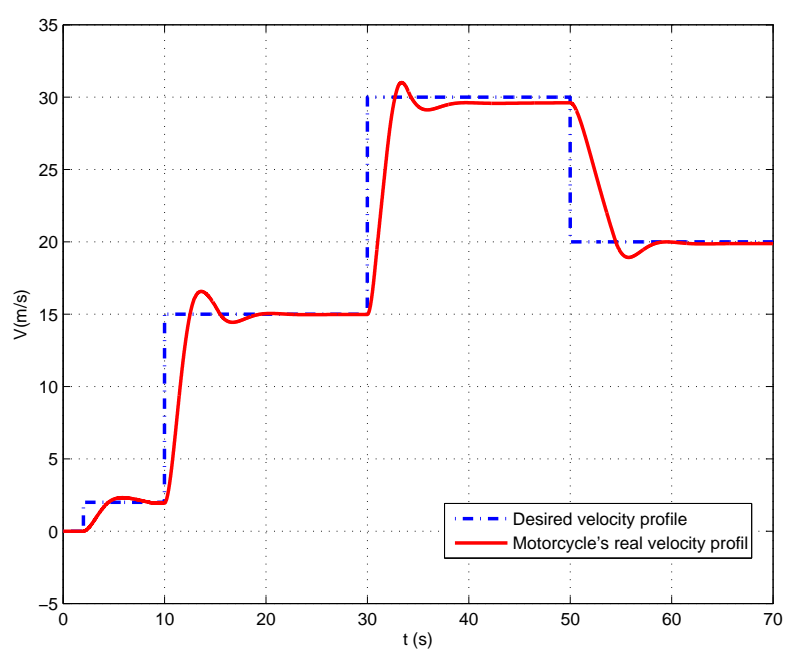

Fig. 7. Motorcycle velocity regulation 


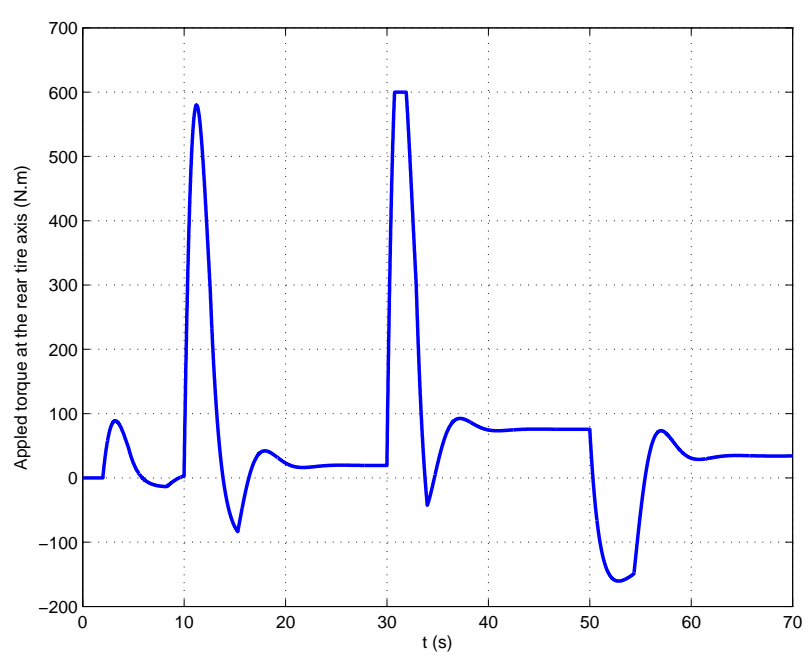

Fig. 8. Rear tire control torque
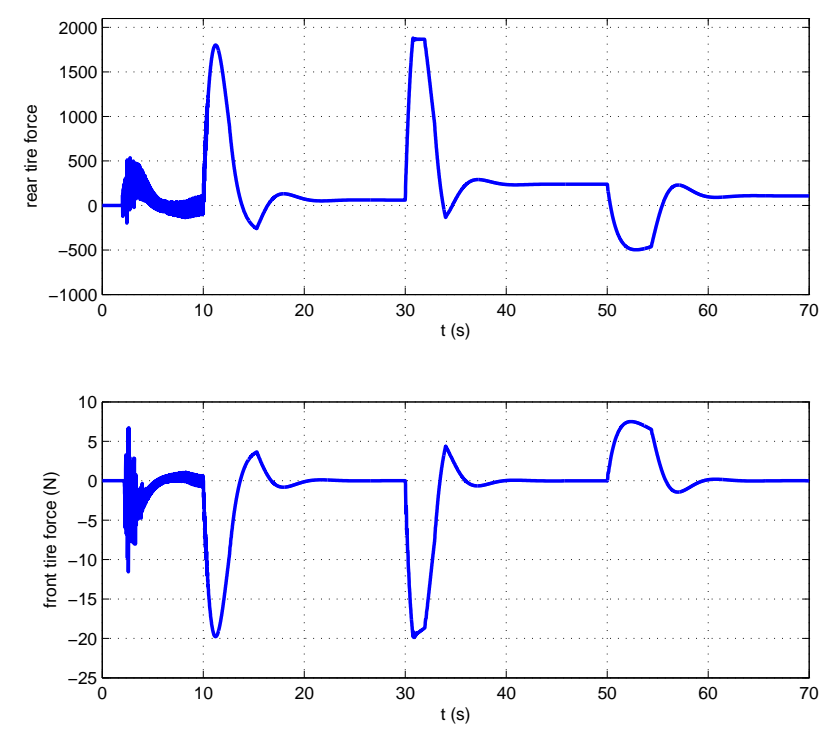

Fig. 9. Rear and front tire/road contact forces

\section{CONCLUSION AND FUTURE WORKS}

In this paper we have proposed a motorcycle dynamics model with a low arithmetic operations number. This model is based on a Newton-Euler recursive algorithm adapted to serial mutlibody chain with floating base. A fast model is needed in some application such as interactive driving simulators, to reduce the delay time between the rider action and the reaction of the motion base platform in order to enhance the rider immersion in the simulation environment.

For the future works, a more global steering controller will be synthesized to evaluate a very complex maneuvers of the synthesized model such as cornering. Besides, this model can be refined by considering the tire/road contact point migration in the tire circumference when cornering, motorcycle engine dynamics model, brakes dynamics model, and finally, a quali- tative evaluation will be done by implementing the synthesized model into our simulator.

\section{REFERENCES}

L. Nehaoua, S. Hima, H. Arioui, N. Seguy and S. Espié Design and Modeling of a New Motorcycle Riding Simulator. American Control Conference, pages 176-181, 2007.

W. Khalil and J. F. Kleinfinger Minimum operations and minimum parameters of the dynamic models of tree structure robots. IEEE Journal on Robotics and Automation, Vol RA3, NO. 6, 1987.

W. Khalil and E. Dombre. Modélisation, identification et commande des robots. Hermes science publications, Paris, 2nd edition, 1999.

J. M. Hollerbach A recursive lagrangian formulation of manipulator dynamics and a comparative study of dynamics formulation complexity. IEEE Transaction on systems, man and cybernetics, vol smc-10, No 11, pages 730-736, 1980.

F. Panerai et al Speed and safety distance control in truck driving: comparison of simulation and real-world environment. Proceedings of driving simulation conference, pages 91-107, 2001.

A. Champion, Ming-Yu Zhang, J. M. Auberlet and S. Espie, Behavioral simulation of a high-density traffic network involving an adaptive ramp metering system. IEEE International Conference on Systems, Man and Cybernetics, vol 5, 2002.

V. Cossalter and R. Lot, A Motorcycle Multi-Body Model for Real Time Simulations Based on the Natural Coordinates Approach. Vehicle System Dynamics, vol 37, No. 6, pages 423-447, 2002.

S. Hima, L. Nehaoua, N. Séguy and H. Arioui, Motorcycle Dynamic Model Synthesis for Two Wheeled Driving Simulator. To appear in International IEEE conference on intelligent transportation systems, 2007.

R. Featherstone and D. Orin, Robot dynamics: equations and algorithms. IEEE International Conference on Robotics and Automation, Vol. 1, pages 826-834, 2000.

A. A. Shabana Dynamics of multibody systems. John wiley \& sons, 1989.

R. Lot, A Motorcycle Tire Model for Dynamic Simulations: Theoretical and Experimental Aspects. Meccanica, an International Journal of Theoretical and Applied Mechanics, Vol. 39, pages 207-220, 2004.

R. S. Sharp and S. Evangelou and D. J. Limebeer, Advances in Modelling of Motorcycle Dynamics. Multibody System Dynamics, Vol. 12, pages 251-283, 2004.

H. B. Pacejka and I. J. M. Besselink, Magic Formulation Tyre Model with Transient Properties. Vehicle System Dynamics, Vol. supplement 27, pages 234-249, 1997. 